\title{
Percepción de influencias en la alimentación de personas mayores del sector Lan-C, comuna de Hualpén, Chile
}

\author{
Claudia Troncoso Pantoja ${ }^{1,2}$, Felipe Echeverría Ferrada ${ }^{3}$, Maximiliano Mellado Arias ${ }^{3}$, Luciano Troncoso Ramírez ${ }^{3}$
}

RESUMEN

Objetivo: Elnterpretar la percepción de las influencias que presenta la alimentación en personas mayores pertenecientes al sector Lan-C de la comuna de Hualpén, Chile, durante el año 2016.

Materiales y métodos: Para el logro de sus metas, la investigación utilizó el paradigma cualitativo con enfoque fenomenológico interpretativo. El muestreo cualitativo se realizó a través del método de bola de nieve. El tamaño de la muestra no se fijó a priori, sino a través del punto de saturación, esto es, cuando las entrevistas no aporten nada nuevo a la investigación. Esta situación se dio en la quinta entrevista. Los participantes que se incluyeron en este estudio cumplieron con los criterios de selección, los que consideraron el ser mayor de 60 años, no presentar problemas cognitivos que impidan desarrollar conversación fluida o diagnóstico de algún tipo de demencia, ser residente del sector Lan-C de la comuna de Hualpén y firmar un consentimiento informado de la investigación. Esto último fue la forma en que se controlaron los aspectos éticos del estudio. La recolección de información se realizó a través de una entrevista semiestructurada. Los resultados fueron codificados y analizados mediante el análisis de contenido.

Resultados: Se nombra por parte de los entrevistados, que su alimentación está influenciada por sus familias, situación económica y emociones. La familia, en especial los hijos, condicionan en forma positiva su alimentación, a través de un apoyo en la selección de alimentos, el tipo de preparaciones a consumir y la mantención o mejoramiento del estado de salud mediante la alimentación. Los factores económicos también son determinantes en su alimentación, mencionando que aunque los ingresos son limitados, se privilegia la adquisición de alimentos sanos que les permitirá mantener una adecuada calidad de vida. En cuanto a las emociones, se aluden especialmente recuerdos de la infancia y juventud, la imagen materna y fechas de conmemoración como condicionantes para su alimentación. Otros factores como la publicidad o las amistades no son identificados como influyentes en su conducta alimentaria.

Conclusiones: En la alimentación de personas mayores, se identifica la percepción de condicionantes que actúan sobre esta, como los factores emocionales, económicos y la influencia familiar.

Palabras clave: Adulto mayor; alimentación; emociones; familia; situación económica.

\section{Perception of influences on the diet of elderly people from the Lan-C sector of Hualpén commune, Chile}

\begin{abstract}
Objective: To interpret the perception of influences on the diet of elderly people from the Lan-C sector of Hualpén commune, Chile, during the year 2016.

Materials and methods: In order to accomplish the objective, researchers used a qualitative design with an interpretative phenomenological approach. The qualitative sampling was performed by the snowball method. The sample size was not determined a priori, but through theoretical saturation; that is, when interviews did not provide new data to the research, which happened in the fifth interview. Subjects included in the study fulfilled the screening criteria, such as being over 60 years old, not having cognitive damage that prevented a fluent conversation, not being diagnosed with any form of dementia, living in the Lan-C sector of Hualpén commune, and signing an informed consent of the research with which ethical aspects of the study were controlled. Data collection was performed through semi-structured interviews. The results were coded and analyzed through the content analysis method.

Results: Interviewees mentioned that their diet was influenced by their family, emotions and economic situation. The family, especially their children, had a positive influence on their diet by selecting the food they were to consume, preparing their meals, and maintaining or improving their health through the diet. They also stated that the economic situation affected their diet and, although they had a limited income, they favored the purchase of healthy food which would allow them to keep an adequate quality of life. Regarding their emotions, they especially refer to memories of their childhood and youth, their mother and special dates which have influence on their diet. Other factors, such as advertisement or friends, were not identified as conditioning their eating behavior.

Conclusion: There are some factors that affect the diet of elderly people, such as their emotions, economic situation and family.
\end{abstract}

Keywords: Elderly people; diet; emotions; family; economic situation.

1. Facultad de Medicina. Departamento de Ciencias Clínicas y Preclínicas. Carrera de Nutrición y Dietética. Universidad Católica de la Santísima Concepción. Chile.

2. Centro de Investigación en Educación y Desarrollo (CIEDE-UCSC). Universidad Católica de la Santísima Concepción. Chile.

3- Carrera de Nutrición y Dietética. Facultad de Medicina. Universidad Católica de la Santísima Concepción. Chile. 


\section{INTRODUCCIÓN}

Una persona no asemeja de la misma manera los sucesos que le ocurren, aceptando o bloqueando estos estímulos con variados métodos. Esta situación tan individual de entender las representaciones mentales de "mi mundo" es lo que se conoce como percepción ${ }^{(1,2)}$, la que puede ser definida como aquella capacidad que presenta la mente de identificar o reconocer estímulos provenientes de los sentidos y que unidos a los recuerdos, interpretan la realidad $^{(3)}$.

En adultos mayores, grupo etario caracterizado por un proceso de envejecimiento demográfico con un incremento exponencial y mantenido en el tiempo de personas mayores de 60 años ${ }^{(4)}$, se presenta una percepción sobre la salud y calidad de vida condicionada por diversos factores, entre los que destacan por ejemplo, la autoestima o la satisfacción con la vida ${ }^{(5)}$. Es por esto que una actitud positiva frente al diario vivir va a depender de ser y estar a gusto consigo mismo ${ }^{(6)}$, así como por el apoyo social y ambiente que les rodea o sus emociones ${ }^{(7)}$. Se ha identificado que las personas mayores que perciben riesgos en su salud, se cuidan más que los que se observan como sanos ${ }^{(8)}$, necesitando tranquilidad que les permita mantener un bienestar idóneo, adecuadas relaciones familiares y la seguridad del hogar así como del poder alimentarse en forma apropiada ${ }^{(9)}$.

La alimentación en las personas mayores puede verse afectada por factores físicos, como alteraciones en la masticación o deglución, entre otros; factores fisiológicos, como una reducción del gasto metabólico basal o alteraciones en el metabolismo de los carbohidratos, en especial, los de absorción rápida y factores psicosociales, como la pobreza o regulares hábitos alimentarios ${ }^{(10)}$.

Los hábitos alimentarios, entendidos como costumbres, actitudes y/o conductas referidas a la alimentación en los adultos mayores, están orientados a mantener o mejorar el estado de salud y calidad de vida de estas personas ${ }^{(11,12)}$, siendo la realidad opuestamente caracterizada por lá presencia de preparaciones no muy variadas, además de presentar a lo menos tres tiempos principales de comidas, el bajo consumo de lácteos, frutas y verduras y el alto consumo de alimentos de elevada densidad energética ${ }^{(13)}$.

En cuanto a la percepción de su alimentación, se identifica una preferencia por preparaciones tradicionales, propias de su cultura, los que les permite fortalecer su identidad ${ }^{(14)}$. Es por esto que la alimentación, específicamente el acto de comer, no solo puede responder al cumplimiento de necesidades biológicas o sociales ${ }^{(15)}$, siendo también una instancia en que estas personas interactúan con la familia 0 amigos ${ }^{(16)}$.

\section{MATERIALES Y MÉTODOS}

Para completar sus metas, la investigación utilizó un diseño cualitativo con enfoque fenomenológico interpretativo, lo que facilita profundizar en la naturaleza de lo que se estudia, mediante la representación de los fenómenos a través de la subjetividad de los pensamientos, permitiendo además, acceder a la conciencia de la persona y explorar las experiencias tal como son vividas o percibidas desde su propia realidad ${ }^{(17,18)}$.

Los sujetos de estudio fueron adultos mayores pertenecientes al sector Lan-C de la comuna de Hualpén, ubicado en la región del Bíobío, provincia de Concepción, Chile, que cumplían con los criterios de selección: ser mayor de 60 años, residente en el sector antes mencionado, sin problemas de cognición, demencia o cualquier patología que limite el desarrollo de un discurso, además de firmar el consentimiento informado.

La investigación profundizó en la percepción que presentan estas personas sobre su alimentación, además de influencias sociales que la condicionan como la familia, amistades, publicidad, situación económica y aspectos emocionales que pueden ser identificados como determinantes para su consumo alimentario.

El muestreo cualitativo utilizado fue de tipo avalancha o bola de nieve, en el que se localiza para este estudio, personas mayores representativos para la investigación, esto es que cumplan con los criterios de selección, a los cuales se les solicita que conduzcan a otros participantes y estos a otros, hasta completar la muestra suficiente para el cumplimiento de las metas del estudio ${ }^{(19)}$.El tamaño de la muestra va a depender únicamente de la riqueza del discurso, que permita profundizar teóricamente en las metas de la investigación, sin fijarse con antelación. En este estudio la muestra se cumplió con cinco personas mayores.

Para la recolección de información, se utilizó una entrevista semiestructurada, la que con anterioridad a su aplicación, fue sometida a validación por expertos. La entrevista fue realizada en forma individual en condiciones establecidas a través de protocolo, en los domicilios particulares de los sujetos de investigación. Esta actividad fue realizada por estudiantes de la carrera de Nutrición y Dietética capacitados por investigador principal.

La conversación individual fue grabada en dispositivo de audio y los resultados transcritos a formato papel para ser codificados e identificar las respectivas categorías para su análisis. Para esto último se utilizó el análisis de contenido.

\section{Aspectos éticos}

El estudio consideró para los aspectos éticos la firma del consentimiento informado, en donde se informa a cada uno de las personas mayores sobre las metas de la investigación, la manera en que se recolectará la información y como se desarrollará su análisis. A lo anterior, se garantiza la privacidad de la persona y su participación voluntaria, además de la posibilidad de abandonar la entrevista en el momento que desee. Se explica que existe una omisión del nombre y los antecedentes generales de la persona al momento de la entrevista y en el desarrollo del informe de la investigación. 


\section{RESULTADOS}

\section{Percepción de la alimentación}

Se identifica, que la importancia en la alimentación para estas personas, radica en variadas motivaciones vinculadas a la percepción, como el permitir sentirse bien o como una oportunidad para el autocuidado, lo que se traduciría finalmente, a opinión de las personas mayores, en mantener una vida más sana.

La totalidad de los entrevistados refieren una relación importante entre la alimentación y su bienestar o salud, considerando el padecimiento de diversas patologías de base; por lo que se vincula esta importancia con el cuidado referente a dichas afecciones, influyendo en la selección de los alimentos a consumir. Se identifican alimentos que no son preferidos debido a la percepción que ellos interpretan para su actual estado de salud: alimentos irritantes, bebidas alcohólicas o el consumo de café.

Las personas mayores reconocen, desde su punto de vista, que su forma de alimentarse es adecuada, debido a que ingieren alimentos que consideran saludables, como frutas o verduras y limitan aquellos que aportan una mayor carga energética, como frituras y grasas. Esta situación también se refleja en la percepción sobre el no presentar limitaciones mayores, especialmente económicas, con respecto a la adquisición de alimentos. También algunos entrevistados interpretan que se alimentan bien, ya que los volúmenes de sus preparaciones son reducidos y acompañados por verduras, además de ser ingeridos en tiempos de alimentación regulares. De esta manera se percibe una recuperación o mantención de una adecuada calidad de vida y salud.

En cuanto a los tiempos de alimentación identificados, una de las entrevistadas menciona en especial al desayuno, describiendo alimentos que codifica como saludables y que por lo demás, los incorpora a su dieta habitual como frutas, avena, yogurt y semillas oleaginosas, percibiendo que estos últimos le favorecen disfrutar una vida más sana. También evita otros alimentos, como el pan, describiéndolo como condicionante para un inadecuado estado de salud, relacionado con la mal nutrición por exceso.

\section{Influencias de la familia en la alimentación}

La familia, señalando los entrevistados en especial a los hijos, son percibidos como un factor positivo en la alimentación de estas personas, debido a que identifican que son ellos, originado por su preocupación filial, los que influencian su consumo de alimentos, direccionando a una alimentación interpretada como saludable, lo que finalmente repercutiría favorablemente en su calidad de vida y estado de salud, beneficio que para más de un entrevistado, se traspasa al grupo familiar.

Solo un participante de la investigación no identifica influencias por parte de su familia en la alimentación, debido a la percepción de que es la entrevistada quien finalmente selecciona su alimentación, respondiendo al cuidado que debe presentar por su cuadro patológico de base, aunque declara que su familia le orienta a seleccionar preparaciones que no compliquen su calidad de vida.

Otro de los entrevistados menciona que realiza acuerdos con su cónyuge para la preparación de sus alimentos, tomando decisiones que le permiten mantener las respectivas preferencias alimentarias, evitando conflictos y manteniendo, desde su pensar, una calidad de vida sana.

\section{Influencia de amistades en la alimentación}

Se interpreta desde el discurso de la totalidad de estas personas mayores, que no se presenta una influencia de su alimentación originada por amistades, entendidas estas últimas como vecinos del sector de residencia.

Se menciona que las opciones en su alimentación son fruto de sus preferencias alimentarias y que sus amistades, a opinión de uno de los entrevistados, no es gravitante, debido a la percepción de que la experiencia adquirida a través de sus vivencias, les permiten tomar sus mejores decisiones.

\section{Influencias de la publicidad en la alimentación}

Las personas mayores no identificarían influencias de la publicidad que condicionen sus preferencias y selección de alimentos, por lo tanto, dentro de su alimentación no son consideradas, al momento de la selección, las sugerencias presentadas por los anuncios publicitarios. Entre las motivaciones que inducen a esta conducta, se establece, desde su perspectiva individual, el conocimiento previo acerca de cómo y qué tipo de alimentos o preparaciones deben consumir de manera adecuada.

\section{Influencias económicas en la alimentación}

Las personas mayores entrevistadas son jubilados de distintas organizaciones de previsión social. Solo dos de los entrevistados reciben ayuda para su alimentación, proveniente de sus hijos.

Los factores económicos también son percibidos como determinantes para su alimentación, esto debido a su reducida capacidad adquisitiva, aunque esto último no es suficiente para acotar la adquisición de alimentos que son considerados como sanos y que les permitirá mejorar su calidad de vida.

Las remuneraciones de estas personas son descritas por todos los entrevistadas como limitadas, por lo que la selección y adquisición de ciertos alimentos se dificulta, pero sin embargo, se realizan esfuerzos o regulan sus gastos para poder ser comprados, con el fin último de una adecuada calidad de vida.

Una de las personas mayores entrevistada reconoce que dentro de su consumo existe presencia de alimentos de elevado costo, causando una dificultad en la compra 
de estos, pero sin llegar a limitar la práctica de una alimentación saludable. Por tanto, prioriza su selección de alimentos y los adapta a su condición económica. Esta última opinión es compartida por los participantes en el estudio.

Además, solo un entrevistado refiere que en instancias de limitación de recursos monetarios, la familia surge como apoyo económico, por lo que no presentaría carencia alimentarias originadas por esta situación.

\section{Influencias emocionales en la alimentación}

Las emociones son importantes y condicionantes para la alimentación de estas personas. En general, se tienden a identificar recuerdos de infancia y juventud, unidos a celebraciones familiares.

De esto último, los entrevistados en su mayoría, relacionan en forma positiva, algunos recuerdos de juventud con fechas de celebración familiar con su alimentación, identificando un tiempo de comida que es específicamente la cena. Declara, uno de los entrevistados, desde su vivencia, que existía un mayor nexo familiar, que les permitía estar unidos en horarios de alimentación, situación ausente actualmente, ya que la familia prefiere o desarrolla tiempos de comidas fuera de su hogar.

También se presenta la percepción de que algunas preparaciones culinarias permiten acercar recuerdos ligados a la imagen de la figura materna y que por lo mismo, actualmente son de mayor preferencia, especialmente algunos platos populares del país y que también les permiten asociar situaciones de alegría, especialmente relacionadas con fechas de conmemoración.

Se presenta, además, una asociación entre sus emociones y estados anímicos, percibiendo una modificación en su ingesta alimentaria cuando se manifiestan variaciones del ánimo, relacionado con el aumento de la frecuencia en el consumo de alimentos.

A lo anterior, se identifica la relación entre la alimentación y el cuidado de su estado de salud, por patologías de base. Los entrevistados declaran y reconocen los beneficios nutricionales que los alimentos les aportan y regulan, con el fin de mantener su calidad de vida.

\section{DISCUSIÓN}

Los estudios realizados en la alimentación de las personas mayores, tiende a centrarse en la cuantificación de los aportes nutritivos que entregan sus dietas, especialmente en personas que están institucionalizadas ${ }^{(20,21)}$. Por el contrario, en adultos mayores ambulatorios, las investigaciones tienden a profundizar en sus preferencias alimentarias, distribución de tiempos y horarios de alimentación, calidad alimentaria y su comportamiento frente a la alimentación, considerando género, condicionantes sociales y económicas para estas personas ${ }^{(22,24)}$.
Esta última situación es la que se presenta en los sujetos investigados, en donde se conciben diversas influencias que entregan las directrices de su alimentación.

En cuanto a la familia, los entrevistados identifican desde distintas perspectivas, algunas influencias en su alimentación, percibiendo en forma positiva esta condicionante. Similar a los resultados encontrados por Vidal et al y Pelcastre et al ${ }^{(25,26)}$, los entrevistados tienden a mencionar que esta influencia familiar es interpretada como importante, ya que está relacionada al cariño o cuidados que le entregan, con el fin último de mantener su estado de salud y calidad de vida, lo que también permite beneficiar a todos los integrantes del grupo familiar.

También en esta investigación, no se menciona la necesidad de depender de algún miembro del grupo familiar para cubrir sus necesidades esenciales, situación que no coincide con los resultados de Vera ${ }^{(27)}$. Sí es percibida la preocupación, especialmente por parte de los hijos, de que reciban una adecuada alimentación que les permita mantener su buen vivir.

En cuanto a los factores económicos que influyen en la alimentación, se identifica que condicionan directamente esta última, aunque en este grupo de personas, no se describe una limitación que afecte directamente su alimentación; en cuanto al acceso, se percibe que afecta más la selección de los alimentos. De esto último, en el artículo dirigido por San Mauro et al ${ }^{(28)}$, se desmitifica la relación entre una alimentación saludable y los costos económicos de esta. En nuestros resultados, los entrevistados identifican limitaciones económicas, pero que sin embargo, no limitan el querer realizar una alimentación saludable, ya que se identifica el deseo de una mejor calidad de vida.

Para este grupo etario, los amigos, como parte de sus redes sociales, tienden a ser identificados como de mediana satisfacción ${ }^{(29)}$, situación que se presenta especialmente en mujeres ${ }^{(30)}$. Esta situación es replicada en nuestros resultados, en cuanto a la influencia que entregan sus amistades, ya que las personas entrevistadas las perciben como no condicionantes para su alimentación.

Otro aspecto en el que se profundizó sobre las influencias en la alimentación de estas personas, fue la publicidad. En nuestro estudio esta no influía en su alimentación y en la selección de sus alimentos, identificando una indiferencia de los entrevistados a esta forma de entrega de información. Esto concuerda con el trabajo realizado por Ramos et al ${ }^{(31)}$ en personas mayores de España, en donde estas últimas, especialmente mujeres, no les agrada la publicidad entregada, la que estaría dirigida a otros grupos etario con imágenes estereotipadas, conservadoras y dirigidas a un segmento específico. Villareal ${ }^{(32)}$ menciona además, que generalmente se liga este grupo etario a desarrollar roles específicos.

En conclusión, el grupo de adultos mayores que participaron en esta investigación, identifican influencias en su alimentación. Algunas condicionantes son percibidas positivamente, 
como en el caso de sus familias, específicamente los hijos. Se identifica, por parte de los entrevistados, que existe una preocupación por el estado de salud que estos presentan, que se traduce hasta en apoyo económico cuando es necesario con algunos participantes, con el fin de que estos adquieran alimentos sanos que se traduzcan en un mejoramiento de su calidad de vida.

Las emociones frente a preparaciones culinarias o algunos alimentos también son percibidos positivamente por los sujetos de estudio, en especial, aquellos platos que son relacionados a recuerdos de infancia o juventud y que se asocian a la unión familiar y/o a la imagen materna. Interpretan que estos producen una reminiscencia que condiciona positivamente su imagen frente a la selección de preparaciones, que tienden a ser platos populares, por lo que les entregan una connotación positiva a la alimentación.

También los aspectos económicos son identificados por estas personas, aunque en sí, no se presenta una percepción negativa sobre este aspecto. Se menciona que los recursos económicos son limitados, pero que sin embargo, esto ha permitido desarrollar una mejor distribución en sus ingresos, lo que facilita la selección de alimentos que son interpretados como saludables.

Otros factores sociales como sus amistades o la publicidad ligada a la alimentación, no condiciona a esta última. Esto puede ser un hallazgo en este estudio, ya que en algunos grupos etario, especialmente niños y jóvenes, estos últimos elementos direccionan considerablemente la selección de alimentos y por lo tanto, su alimentación.

A lo anterior y otro hallazgo de esta investigación es que las personas mayores que participaron, pueden identificar claramente desde un punto de vista nutricional, los alimentos que les son perjudiciales para su calidad de vida, especialmente aquellos que aportan lípidos de origen animal o la relación que presenta el volumen de ingesta alimentaria con la presencia de malnutrición por exceso. También reconocen y mencionan aquellos alimentos que aportan nutrientes necesarios para este grupo etario, como el calcio, por ejemplo.

Esto último puede llevarnos a la reflexión, como agentes del área de salud. Se tiende a subestimar el nivel de conocimiento que las personas mayores pueden presentar sobre el cuidado en su salud a través de la alimentación. En nuestro estudio las personas entrevistadas tienen una concepción adecuada desde un punto de vista nutricional, sobre una alimentación saludable, la que se manifiesta a través de las preparaciones culinarias consumidas, con el fin de mantener su estado de salud y por lo mismo, su calidad de vida.

Una limitante en esta investigación es lo acotado del sector en donde se obtuvieron los resultados, lo que pudo haber favorecido la rápida saturación teórica. Sin embargo, también puede abrir una veta para profundizar más en la limitada importancia que perciben las personas mayores sobre la influencias en su alimentación.
Entonces es importarse seguir profundizando en las conductas alimentarias en personas mayores desde diversos paradigmas o estrategias, lo que permitirá fortalecer la opinión de personas mayores en aspectos de la alimentación, más allá que la entregada a través de un trabajo con diseño cualitativo, o para mayor enriquecimiento complementar con otro tipo de diseño.

Agradecimiento: Se agradece a todas las personas que permitieron el desarrollo de este estudio, en especial, los vecinos del sector Lan-C.

\section{REFERENCIAS BIBLIOGRÁFICAS}

1. Durand L. De las percepciones a las perspectivas ambientales. Una reflexión teórica sobre la antropología y la temática ambiental. Obtenido en agosto de 2016. Disponible en: http:// historico.juridicas.unam.mx/publica/librev/rev/nuant/ cont/68/pr/pr5.pdf

2. Oviedo G. La definición del concepto de percepción en psicología con base en la Teoría Gestalt. rev. estud. soc. 2004; 18:89-96.

3. Sánchez I, Pérez V. El funcionamiento cognitivo en la vejez: atención y percepción en el adulto mayor. Rev Cubana Med Gen Integr. 2008; 24(2): 1-7.

4. Betancourt C. Envejecimiento demográfico y necesidad de desarrollar las competencias profesionales en enfermería geriátrica. Rev haban cienc méd. 2015; 14(1): 89-96.

5. Vivaldi F, Barra E. Bienestar psicológico, apoyo social percibido y percepción de salud en adultos mayores. Ter Psicol. 2012; 30(2): 23-29.

6. Zavala M, Vidal D, Castro $M$ et al. Funcionamiento social del adulto mayor. Cienc. enferm. 2006; 12(2): 53-62.

7. Fernández R, Manrique F. Rol de la enfermería en el apoyo social del adulto mayor. Enfermería Global. 2010; 9(2):1-9.

8. Millán I. Evaluación de la capacidad y percepción de autocuidado del adulto mayor en la comunidad. Rev Cubana Enfermer. 2010; 26(4): 202-34.

9. Vera M. Significado de la calidad de vida del adulto mayor para sí mismo y para su familia. An Fac med. 2007; 68(3): 284-90.

10. Varela L. Nutrición en el adulto mayor. Rev Med Hered. 2013; 24(3): $1-2$.

11. Unicef. Formación de hábitos alimentarios y de estilos de vida saludables. Obtenido en agosto de 2016. Disponible en: http:// www.unicef.org/venezuela/spanish/educinic9.pdf

12. Silvestri C. Hábitos alimentarios en relación al consumo de alimentos protectores de la salud en adultos mayores de más de 70 años. Obtenido en agosto de 2016.

Disponible en: http://imgbiblio.vaneduc.edu.ar/fulltext/files/ TC111529.pdf

13. Restrepo S, Morales R, Ramírez M et al. Los hábitos alimentarios en el adulto mayor y su relación con los procesos protectores y deteriorantes en salud. Rev Chil Nutr. 2006; 33(3):500-10.

14. Franco F, Arboleda L. Aspectos socioculturales y técnicosnutricionales en la alimentación de un grupo de adultos mayores del centro gerontológico Colonia de Belencito de Medellín-Colombia. Perpesct Nutr Humana. 2010; 12(1): 61-74.

15. Próspero 0 , Méndez $M$, Alvarado I et al. Inteligencia para la alimentación: alimentación para la inteligencia. Salud Mental. 2013; 36 (2):101-7.

16. Hernández Z, Hernández 0 , Rodríguez E. El grupo de ayuda 
como alternativa para mejorar la calidad de vida del adulto mayor. Psicología Iberoamericana. 2010; 18(2): 47-55.

17. Trejo F. Fenomenología como método de investigación: Una opción para el profesional de enfermería. Enf Neurol (Mex). 2012; $11(2)$ : 98-101.

18. Guerrero R, Ojeda M. La fenomenología y su uso en la producción científica de enfermería: estudio bibliométrico 2010-2014. Ra Ximhai. 2015; 11(2): 193-206.

19. Salamanca AB, Martín-Crespo C. El muestreo en la investigación cualitativa. Nure investigación [Internet]. 2007 [citado 07 de marzo 2017]; 27:1-4. Disponible en: http:// www. nureinvestigacion.es/OJS/index.php/nure/article/ view/340/330

20. Sánchez M, Torralba C, López M, Zamora S, Perez-Llamas F. Estrategias para mejorar el valor nutricional de los menús ofertados en residencias públicas para personas mayores. Nutr. Hosp. 2010;25(6): 1014-1019.

21. Dudet M. Valoración nutricional de la oferta y de la ingesta dietética en una residencia geriátrica. Rev Esp Nutr Comunitaria. 2013; 19(1): 20-8.

22. García P, Durán S. Asociación del consumo de desayuno y la calidad de vida en adultos mayores autónomos chilenos. Nutr Hosp. 2014; 30(4): 845-50.

23. Hernández A, Pontes $Y$, Goñi I. Riesgo de malnutrición en una población mayor de 75 años no institucionalizada con autonomía funcional. Nutr Hosp. 2015; 32(3): 1184-92.

24. Hernández A, Goñi I. Calidad de la dieta de la población española mayor de 80 años no institucionalizada. Nutr Hosp. 2015; 31(6): 2571-7.

25. Vida $D$, Zavala $M$, Castro $M$ et al. Calidad de vida en el adulto mayor: estado físico y relaciones sociales. Cuad Méd Soc (Chile). 2008; 48(1): 5-12.

26. Pelcastre B, Treviño S, González $T$ et al. Apoyo social y condiciones de vida de adultos mayores que viven en la pobreza urbana en México. Cad. Saúde Pública. 2011; 27(3): 460-470.

27. Vera M. Significado de la calidad de vida del adulto mayor para sí mismo y para su familia. An. Fac. med. 2007; 68(3): 284-290.

28. Sam Mauro I, Cendón M, Soulas C et al. Planificación alimentaria en personas mayores: aspectos nutricionales. Nutr Hosp. 2012; 27(6): 2116-2121

29. Cardona D, Estrada A, Chavarriaga L et al. Apoyo social dignificante del adulto mayor institucionalizado. Medellín, 2008. Rev. Salud pública. 2010; 12(3): 414-424.

30. Zapata B, Delgado N, Cardona D. Apoyo social y familiar al adulto mayor del área urbana en Angelópolis, Colombia 2011. Rev. salud pública. 2015; 17(6): 848-860.

31. Ramos I, Caretón M. Presencia y representación de las personas mayores en la publicidad televisiva: el caso español. Rev Esp Geriatr Gerontol. 2012; 47(2): 55-61.

32. Villareal A. Publicidad televisiva reproduce cautiverios femeninos. Revista Escena. 2010; 33(66): 137-154.

Fuentes de financiamiento:

Este artículo ha sido financiado por los autores.

Conflictos de interés:

Los autores declaran no tener ningún conflicto de interés.

\section{Correspondencia:}

Claudia Troncoso Pantoja

Dirección: Universidad Católica de la Santísima Concepción.

Facultad de Medicina.

Campus San Andrés. Alonso de Ribera 2850. Concepción, Chile.

Teléfono: 56-041-2345427 - 56-041-2345406

Correo electrónico: ctroncosop@ucsc.cl

Recibido: 05 de setiembre de 2016 Aprobado: 25 de noviembre de 2016 